\title{
DETERMINATION OF MINIMUM LENGTH OF SOME LINEAR CODES
}

\author{
EUN Ju CHEON*
}

\begin{abstract}
Hamada ([8]) and Maruta ([17]) proved the minimum length $n_{3}(6, d)=g_{3}(6, d)+1$ for some ternary codes. In this paper we consider such minimum length problem for $q \geq 4$, and we prove that $n_{q}(6, d)=g_{q}(6, d)+1$ for $d=q^{5}-q^{3}-q^{2}-2 q+e, 1 \leq e \leq q$. Combining this result with Theorem $\mathrm{A}$ in [4], we have $n_{q}(6, d)=$ $g_{q}(6, d)+1$ for $q^{5}-q^{3}-q^{2}-2 q+1 \leq d \leq q^{5}-q^{3}-q^{2}$ with $q \geq 4$. Note that $n_{q}(6, d)=g_{q}(6, d)$ for $q^{5}-q^{3}-q^{2}+1 \leq d \leq q^{5}$ by
\end{abstract} Theorem 1.2.

\section{Introduction}

Let $\mathbb{F}_{q}$ denote the Galois field of $q$ elements and $\mathbb{F}_{q}^{n}$ denote the $n$ dimensional vector space over $\mathbb{F}_{q}$, where $q$ is a prime power. For a vector $x=\left(x_{1}, \ldots, x_{n}\right) \in \mathbb{F}_{q}^{n}$, the weight of $x$ denoted by $w(x)$ is the number of nonzero coordinates of $x$, that is, $w(x)=\left|\left\{i \mid x_{i} \neq 0\right\}\right|$.

An $[n, k, d]_{q}$ linear code $C$ is a $k$-dimensional subspace of $\mathbb{F}_{q}^{n}$ over $\mathbb{F}_{q}$ with minimum distance $d$. One of the central problems in coding theory is to determine optimal linear codes. This is to optimize one of the parameters $n, k$ and $d$ for given the other two as follows; (1) Find the smallest length $n$, denoted by $n_{q}(k, d)$, for which there exists an $[n, k, d]_{q}$ code for given $k$ and $d$. (2) Find the largest minimum distance $d$, denoted by $d_{q}(n, k)$, for which there exists an $[n, k, d]_{q}$ code for given $k$ and $n$. (3) Find the largest dimension $k$, denoted by $k_{q}(n, d)$, for which there exists an $[n, k, d]_{q}$ code for given $n$ and $d$.

Received October 17, 2012; Accepted January 11, 2013.

2010 Mathematics Subject Classification: Primary 94B65, 94B05, 51E20, 05B25.

Key words and phrases: linear code, minimum length, Griesmer bound, minihyper, projective space.

This work was supported by Basic Science Research Program through the National Research Foundation of Korea(NRF) funded by the Ministry of Education, Science and Technology(MEST)(2012-0004252). 
A code of length $n_{q}(k, d)$ [resp. minimum distance $d_{q}(n, k)$, dimension $\left.k_{q}(n, d)\right]$ is said to be length-optimal [resp. distance-optimal, dimensionoptimal]. Note that a length-optimal code is both distance-optimal and dimension-optimal. So we concentrate on the length-optimal codes. The following is an important lower bound on $n_{q}(k, d)$ which is called the Griesmer bound.

Theorem $1.1([9])$. (Griesmer bound) For an $[n, k, d]_{q}$ linear code, we have $n \geq g_{q}(k, d)$, where $g_{q}(k, d)=d+\left\lceil\frac{d}{q}\right\rceil+\left\lceil\frac{d}{q^{2}}\right\rceil+\cdots+\left\lceil\frac{d}{q^{k-1}}\right\rceil$.

By Theorem 1.1, we note $n_{q}(k, d) \geq g_{q}(k, d)$ for all $k$ and $d$. It is natural to ask whether there exists a $\left[g_{q}(k, d), k, d\right]_{q}$ code for given $d$ and $k$. The following theorem gives a large class of linear codes meeting the Griesmer bound which we call Griesmer codes.

Theorem $1.2([9])$. Let $s=\left\lceil\frac{d}{q^{k-1}}\right\rceil$ and $d=s q^{k-1}-\sum_{i=1}^{p} q^{u_{i}-1}$ with $k>u_{1} \geq u_{2} \geq \cdots \geq u_{p}$ and $u_{i}>u_{i+q-1}$ for $1 \leq i \leq p-q+1$. If

$$
\sum_{i=1}^{\min \{s+1, p\}} u_{i} \leq s k,
$$

then $n_{q}(k, d)=g_{q}(k, d)$.

Theorem 1.2 provides a starting point for finding $n_{q}(k, d)$. For $k=1$ and 2 , we have $n_{q}(k, d)=g_{q}(k, d)$ for all $d$. Thus we are interested in $k \geq 3$.

From Theorem 1.2, we have the following:

Corollary $1.3([5])$. We have $n_{q}(k, d)=g_{q}(k, d)$ for $d$ satisfying one of the following:

(a) $q^{k-1}-q^{k-1-t}-q^{t}+1 \leq d \leq q^{k-1}-q^{k-1-t}$

$$
\text { with } 1 \leq t \leq\left\lfloor\frac{k-1}{2}\right\rfloor-1 \text { for } k \geq 5 \text {, }
$$

(b) $q^{k-1}-q^{k-1-t}-q^{t}+1 \leq d \leq q^{k-1}$ with $t=\left\lfloor\frac{k-1}{2}\right\rfloor$ for $k \geq 3$,

where $\lfloor x\rfloor$ denotes the largest integer less than or equal to $x$.

Much research on $n_{q}(k, d)$ has been done for small dimension $k$ and small $q$ by various methods. For $k=3,4,5$ and $q=3,4,5$, we can find tables of the values of $n_{q}(k, d)$ in [11] and [16].

To find the value of $n_{q}(k, d)$ for general $q$ or $k$ is more interesting. For minimum distance $d$ with $q^{k-1}-q^{k-1-t}-q^{t}-s q+1 \leq d \leq q^{k-1}-$ 
$q^{k-1-t}-q^{t}-(s-1) q$ for $1 \leq t \leq\left\lfloor\frac{k-1}{2}\right\rfloor$ and $1 \leq s \leq q-1$, which is just below the values for $d$ in Corollary 1.3, it is known that there is no Griesmer code with

(1) $t=1$ and $s=1$ for $q \geq 3, k \geq 5$ in $[3,4,13,14,15]$,

(2) $t=1$ and $2 \leq s \leq q-1$ in [15],

(3) $s=1$ and $t=\left\lfloor\frac{k-1}{2}\right\rfloor$ for $k \geq 5$ in [3], $2 \leq t \leq\left\lfloor\frac{k-1}{2}\right\rfloor-1$ for $k \geq 7$ in [5].

Naturally we can ask the cases $t \geq 2$ or $s \geq 2$. When $t=2$ and $s=2$, there is a result for the question for $k=5$ in [2].

In this paper, we consider the case $t=2$ and $s=2$ for $k=6$. In other words, we consider the problem whether Griesmer codes with minimum distance $d$ with $q^{5}-q^{3}-q^{2}-2 q+1 \leq d \leq q^{5}-q^{3}-q^{2}-q$ exist or not for $q \geq 4$. For $q=2$ or 3 , we note that $n_{2}(6, d)=g_{2}(6, d)$ with $d=17,18$ $([6])$ and $n_{3}(6, d)=g_{3}(6, d)+1$ with $d=202,203,204([8,17])$.

As the first step to determine the exact value of $n_{q}(6, d)$ with $d=$ $q^{5}-q^{3}-q^{2}-2 q+\alpha, 1 \leq \alpha \leq q$ and $q \geq 4$, we need to prove the following.

Theorem A. There does not exist a $\left[g_{q}(6, d), 6, d\right]_{q}$ code with $d=$ $q^{5}-q^{3}-q^{2}-2 q+1$ for $q \geq 4$.

In Section 3, we give a proof of Theorem A and in Section 2, we recall some results needed to prove Theorem A.

Recall that the existence of an $[n, k, d]_{q}$ code with $d \geq 2$ implies the existence of an $[n-1, k, d-1]_{q}$ code. Therefore, by Theorem A, we have the following.

Theorem B. For $q \geq 4$, we have $n_{q}(6, d) \geq g_{q}(6, d)+1$ with $q^{5}-$ $q^{3}-q^{2}-2 q+1 \leq d \leq q^{5}-q^{3}-q^{2}-q$.

If we let $k=6$ in Theorem 16 in [3], then we have the following.

Theorem $1.4([3])$. For $q \geq 3$, there exists a $\left[g_{q}(6, d)+1,6, d\right]_{q}$ code for $q^{5}-q^{3}-2 q^{2}+1 \leq d \leq q^{5}-q^{3}-q^{2}$.

By Theorem B and Theorem 1.4, we conclude the next theorem.

Theorem C. For $q \geq 4$, we have $n_{q}(6, d)=g_{q}(6, d)+1$ with $q^{5}-$ $q^{3}-q^{2}-2 q+1 \leq d \leq q^{5}-q^{3}-q^{2}-q$.

Finally, combining the result of [4] with Theorem $\mathrm{C}$, for $q \geq 4$, we have the following:

$$
n_{q}(6, d)=g_{q}(6, d)+1 \text { for } q^{5}-q^{3}-q^{2}-2 q+1 \leq d \leq q^{5}-q^{3}-q^{2} .
$$




\section{Preliminaries}

Let $P G(r, q)$ be the $r$-dimensional projective space over $\mathbb{F}_{q}$ and let $\theta_{r}$ be the number of points in $P G(r, q)$. Then $\theta_{r}=q^{r}+q^{r-1}+\cdots+q+1$ for a positive integer $r$. For convenience, we let $\theta_{0}=1$ and $\theta_{r}=0$ if $r<0$. We call a subspace of dimension $j$ in $P G(r, q)$ a $j$-flat. In particular, we call a subspace of dimension 0 [resp. 1, 2, r-1] a point [resp. a line, a plane, a hyperplane].

Let $C$ be a projective $[n, k, d]_{q}$ linear code with a generator matrix of $G$. Then no two columns of $G$ are linearly dependent. Each column of $G$ can be considered as a point of $P G(k-1, q)$. Let $C_{1}$ be the set of all columns of $G$ and let $C_{0}=C_{1}^{c}$, the complement of $C_{1}$ in $P G(k-1, q)$. For a subset $S$ in $P G(k-1, q)$, we use the following notation;

$$
c_{0}(S)=\left|S \cap C_{0}\right|, \quad c(S)=\left|S \cap C_{1}\right| \quad \text { and } \quad c_{0}=\left|C_{0}\right| .
$$

In particular, for a projective $[n, 6, d]_{q}$ linear code $C$, we have $n=$ $\left|C_{1}\right|, c_{0}=\theta_{5}-n$ and $d=n-\max \{c(H) \mid H$ is a 4-flat in $P G(5, q)\}$.

Now we recall theorems which play an important role to prove Theorem A.

For a subset $S$ in the $r$-dimensional affine space $A G(r, q)$ over $\mathbb{F}_{q}, S$ is a $t$-fold blocking set with respect to hyperplanes if every hyperplane in $A G(r, q)$ meets $S$ in at least $t$ points.

TheOREM 2.1 ([1]). A $t$-fold blocking set $S$ with respect to hyperplanes in $A G(r, q)$ satisfies

$$
|S| \geq(r+t-1)(q-1)+1 .
$$

A subset $F$ of $P G(r, q)$ with $|F|=f$ is called an $\{f, t ; r, q\}$-minihyper if every hyperplane meets $F$ in at least $t$ points. Hamada ([7]) showed that for $k \geq 3$ and $1 \leq d<q^{k-1}$, there is a one to one correspondence between the set of all nonequivalent $[n, k, d]_{q}$ Griesmer codes and the set of all $\left\{\theta_{k-1}-n, \theta_{k-2}-n+d ; k-1, q\right\}$-minihypers. Thus an $[n, 6, d]_{q}$ Griesmer code $C$ with $d<q^{5}$ corresponds to a $\left\{\theta_{5}-n, \theta_{4}-n+d ; 5, q\right\}$ minihyper. The following is a characterization of some minihypers.

Theorem $2.2([7])$. Let $\lambda_{1}, \lambda_{2}, \ldots, \lambda_{m}$ be positive integers with $1 \leq$ $\lambda_{1}<\lambda_{2}<\cdots<\lambda_{m} \leq t$ and $1 \leq m \leq t$.

(a) In the case $m=1, S$ is a $\left\{\theta_{\lambda_{1}}, \theta_{\lambda_{1}-1} ; t, q\right\}$-minihyper if and only if $S$ is a $\lambda_{1}$-flat in $P G(t, q)$.

(b) In the case $m \geq 2$ and $t \geq \lambda_{m}+\lambda_{m-1}+1$, $S$ is a $\left\{\sum_{i=1}^{m} \theta_{\lambda_{i}}, \sum_{i=1}^{m} \theta_{\lambda_{i}-1}\right.$; $t, q\}$-minihyper if and only if $S$ consists of disjoint union of $\lambda_{i}$-flats in $P G(t, q)$. 
(c) In the case $m \geq 2$ and $t \leq \lambda_{m}+\lambda_{m-1}$, there is no $\left\{\sum_{i=1}^{m} \theta_{\lambda_{i}}\right.$, $\left.\sum_{i=1}^{m} \theta_{\lambda_{i}-1} ; t, q\right\}$-minihyper.

Let $m_{r, q}(s)$ denote the minimum value of $f$ for which an $\left\{f, \theta_{r-2}+\right.$ $s ; r, q\}$-minihyper exists for $r \geq 3$ and $1 \leq s \leq q-1$. If we let $r=4$ and $s=1$ or $s=2$ in Theorem 2.4 in [15], then we have the following.

Theorem $2.3([15])$. For $q \geq 3$, we have

(a) $m_{4, q}(1) \geq \theta_{3}+\theta_{1}+q$,

(b) $m_{4, q}(2) \geq \theta_{3}+2 \theta_{1}+q$.

In [14], Maruta proved the nonexistence of a $\left[g_{q}(5, d), 5, d\right]_{q}$ code with $q^{4}-2 q^{2}-q+1 \leq d \leq q^{4}-2 q^{2}$ for $q \geq 3$. Landjev and Maruta [resp. Cheon et al.] proved the nonexistence of a $\left[g_{q}(5, d), 5, d\right]_{q}$ code with $q^{4}-2 q^{2}-2 q+1 \leq d \leq q^{4}-2 q^{2}-q$ for $q=4$ [resp. for $q \geq 5$ ] in [12] [resp. [2]]. Since those two intervals of $d$-values are consecutive, we conclude that there does not exist a Griesmer code with $q^{4}-2 q^{2}-2 q+1 \leq$ $d \leq q^{4}-2 q^{2}$ for $q \geq 4$. Here we express the above results with the notion of minihyper respectively.

Theorem 2.4 ([14]). For $q \geq 3$ and $0 \leq e \leq q-1$, there does not exist a $\left\{2 \theta_{2}+e, 2 \theta_{1} ; 4, q\right\}$-minihyper.

Theorem 2.5 ([2, 12]). For $q \geq 4$ and $0 \leq e \leq q-1$, there does not exist a $\left\{2 \theta_{2}+\theta_{1}+e, 2 \theta_{1}+1 ; 4, q\right\}$-minihyper.

For a Griesmer code, the following holds:

Theorem $2.6([14])$. Let $C$ be a $\left[g_{q}(k, d), k, d\right]_{q}$ code and let $\gamma_{j}:=$ $\sum_{i=0}^{j}\left\lceil\frac{d}{q^{k-1-i}}\right\rceil$ for $0 \leq j \leq k-2$. Then there exist $j$-flats $\Delta_{j}$ with $c\left(\Delta_{j}\right)=\gamma_{j}$ such that $\Delta_{0} \subseteq \Delta_{1} \subseteq \cdots \subseteq \Delta_{k-2}$ and that $\Delta_{j}$ gives a $\left[\gamma_{j}, j+1, \gamma_{j}-\gamma_{j-1}\right]$ Griesmer code for $1 \leq j \leq k-2$.

If we let $t=1$ in Theorem 7 in [10], then we have the following.

Theorem 2.7. For an integer $r \geq 0$ and $q \geq r+1$, let $S$ be a subset in $P G(m, q)$ with $|S| \leq \theta_{m-1}+r \theta_{m-3}$. If $|S \cap l| \geq 1$ for any line $l$ in $P G(m, q)$ then $S$ contains a hyperplane.

\section{Main theorem}

In this section, we prove Theorem A. On the contrary, we assume that for $q \geq 4$, there exists a $\left[g_{q}(6, d), 6, d\right]_{q}$ code $C$ with $d=q^{5}-q^{3}-q^{2}-2 q+1$. 
Since $C$ is a Griesmer code, by Theorem 2.6, we have the following:

$$
\begin{aligned}
& c_{0}=\theta_{3}+\theta_{2}+2 q, \\
& c_{0}(H) \geq \theta_{2}+\theta_{1}+1 \text { for any } 4 \text {-flat } H \text { in } P G(5, q), \\
& c_{0}(\Delta) \geq \theta_{1}+1 \text { for any } 3 \text {-flat } \Delta \text { in } P G(5, q), \\
& c_{0}(\delta) \geq 1 \text { for any 2-flat } \delta \text { in } P G(5, q) .
\end{aligned}
$$

Let $H_{0}$ be a 4-flat in $P G(5, q)$ with $c_{0}\left(H_{0}\right)=\theta_{2}+\theta_{1}+1$. Then $H_{0}$ is a $\left\{\theta_{2}+\theta_{1}+1, \theta_{1}+1 ; 4, q\right\}$-minihyper. By Theorem $2.2(\mathrm{~b})$, we note that $H_{0} \cap C_{0}$ is a disjoint union of a plane $\delta_{0}$, a line $l_{0}$ and a point $P_{0}$. For any 3 -flat $\Delta$ in $H_{0}$, we have

(3.1) $c_{0}(\Delta)=\theta_{2}+2, \quad \theta_{2}+1, \quad 2 \theta_{1}+1, \quad 2 \theta_{1}, \quad \theta_{1}+2, \quad$ or $\quad \theta_{1}+1$.

For any 4-flat $H$ in $P G(5, q)$, we have $c_{0}\left(H_{0} \cap H\right) \leq \theta_{2}+2$ by (3.1). Since $c_{0}(H) \geq \theta_{2}+\theta_{1}+1$ for any 4 -flat $H$, we have

$$
\begin{aligned}
c_{0} & =c_{0}(H)+\sum_{H_{0} \cap H \subseteq H^{\prime} \neq H} c_{0}\left(H^{\prime}\right)-q c_{0}\left(H_{0} \cap H\right) \\
& \geq c_{0}(H)+q\left(\theta_{2}+\theta_{1}+1\right)-q\left(\theta_{2}+2\right) \\
& =c_{0}(H)+q^{2},
\end{aligned}
$$

which implies $c_{0}(H) \leq \theta_{3}+2 \theta_{1}+q-1$.

Therefore, we conclude that

(3.2) $\theta_{2}+\theta_{1}+1 \leq c_{0}(H) \leq \theta_{3}+2 \theta_{1}+q-1$ for any 4-flat $H$ in $P G(5, q)$.

Now we will derive a contradiction in two steps as follows: In Step I, we prove that there is no 4 -flat $H$ such that

$$
2 \theta_{2}+1 \leq c_{0}(H) \leq \theta_{3}+2 \theta_{2}+q-1 .
$$

Then, by (3.2), we conclude that $\theta_{2}+\theta_{1}+1 \leq c_{0}(H) \leq 2 \theta_{2}$ for any 4-flat $H$ in $P G(5, q)$. In Step II, we will prove that it is impossible.

Step I. We divide the interval (3.3) into five small intervals, which we refer to as Case $1, \ldots$, Case 5 and we prove the nonexistence of a 4 -flat $H$ with $c_{0}(H)$ belonging to each small interval.

When we prove them we use the following computation frequently: For a 4 -flat $H_{1}$ in $P G(5, q)$, let $\Delta$ be a 3 -flat in $H_{1}$. Then we have

$$
\begin{aligned}
c_{0} & =c_{0}\left(H_{1}\right)+\sum_{\Delta \subseteq H \neq H_{1}} c_{0}(H)-q c_{0}(\Delta) \\
& \geq c_{0}\left(H_{1}\right)+q\left(\theta_{2}+\theta_{1}+1\right)-q c_{0}(\Delta),
\end{aligned}
$$


and hence

$$
c_{0}(\Delta) \geq \frac{c_{0}\left(H_{1}\right)-c_{0}}{q}+\theta_{2}+\theta_{1}+1
$$

Case 1. There is no 4-flat $H$ with $\theta_{3}+2 \theta_{1} \leq c_{0}(H) \leq \theta_{3}+2 \theta_{1}+q-1$.

Proof. Suppose that there exists a 4 -flat $H_{1}$ with $c_{0}\left(H_{1}\right)=\theta_{3}+2 \theta_{1}+$ $q-1-f, 0 \leq f \leq q-1$. Let $\Delta$ be a 3 -flat in $H_{1}$. Using (3.4), we have $c_{0}(\Delta) \geq \theta_{2}+2-\frac{f}{q}$. Since $0 \leq f \leq q-1$, we have

$$
c_{0}(\Delta) \geq \theta_{2}+2 \text { for all } 3 \text {-flat } \Delta \subset H_{1} .
$$

Furthermore, if we let $\Delta=H_{0} \cap H_{1}$, then $c_{0}(\Delta)=\theta_{2}+2$ since $c_{0}\left(H_{0} \cap H_{1}\right) \leq \theta_{2}+2$ by (3.1). Thus $C_{0} \cap H_{1}$ is a $\left\{\theta_{3}+2 \theta_{1}+q-1-\right.$ $\left.f, \theta_{2}+2 ; 4, q\right\}$-minihyper, which contradicts Theorem 2.3 (b).

Case 2. There is no 4-flat $H$ with $\theta_{3}+\theta_{1}+1 \leq c_{0}(H) \leq \theta_{3}+\theta_{1}+q$.

Proof. Suppose that there exists a 4 -flat $H_{1}$ with $c_{0}\left(H_{1}\right)=\theta_{3}+\theta_{1}+$ $q-f, 0 \leq f \leq q-1$. Let $\Delta$ be a 3-flat in $H_{1}$. Using (3.4), we have $c_{0}(\Delta) \geq \theta_{2}+1$ since $0 \leq f \leq q-1$.

Suppose that $c_{0}(\Delta) \geq \theta_{2}+2$ for all 3-flat $\Delta \subseteq H_{1}$. By Theorem 2.3 (b), we have $c_{0}\left(H_{1}\right) \geq \theta_{3}+2 \theta_{1}+q$ which is a contradiction. Thus there exists a 3 -flat $\Delta$ with $c_{0}(\Delta)=\theta_{2}+1$. Then $C_{0} \cap H_{1}$ is a $\left\{\theta_{3}+\theta_{1}+q-\right.$ $\left.f, \theta_{2}+1 ; 4, q\right\}$-minihyper. By Theorem 2.3 (a), we obtain $f=0$, that is, $C_{0} \cap H_{1}$ is a $\left\{\theta_{3}+\theta_{1}+q, \theta_{2}+1 ; 4, q\right\}$-minihyper. Here, we prove the following claim.

Claim. $C_{0} \cap H_{1}$ contains a 3-flat.

Proof of Claim: To prove Claim, it suffices to prove that for any line $l$ in $H_{1},\left|\left(C_{0} \cap H_{1}\right) \cap l\right| \geq 1$ by Theorem 2.7. Suppose that there is a line $l_{1}$ in $H_{1}$ with $\left|\left(C_{0} \cap H_{1}\right) \cap l_{1}\right|=0$. Consider a 3 -flat $\Delta$ in $H_{1}$ containing $l_{1}$. Then there is a 3 -flat $\Delta^{\prime}$ containing $l_{1}$ with $\left|\left(C_{0} \cap H_{1}\right) \cap \Delta^{\prime}\right|=\theta_{2}+1$ since $c_{0}\left(H_{1}\right)=\theta_{3}+\theta_{1}+q$ and

$$
c_{0}\left(H_{1}\right)=\frac{1}{\theta_{1}}\left(\sum_{l_{1} \subset \Delta \subset H_{1}}\left|\left(C_{0} \cap H_{1}\right) \cap \Delta\right|\right)<\frac{1}{\theta_{1}} \cdot \theta_{2} \cdot\left(\theta_{2}+2\right) .
$$

On the other hand, we have

$$
\begin{aligned}
c_{0} & =c_{0}\left(H_{1}\right)+\sum_{\Delta^{\prime} \subseteq H, H \neq H_{1}} c_{0}(H)-q c_{0}\left(\Delta^{\prime}\right) \\
& \geq c_{0}\left(H_{1}\right)+q\left(\theta_{2}+\theta_{1}+1\right)-q\left(\theta_{2}+1\right)=\theta_{3}+\theta_{2}+2 q .
\end{aligned}
$$


Thus we note $c_{0}(H)=\theta_{2}+\theta_{1}+1$ for any 4 -flat $H\left(\neq H_{1}\right)$ containing $\Delta^{\prime}$. Let $H_{2}$ be a 4 -flat containing $\Delta^{\prime}$ with $c_{0}\left(H_{2}\right)=\theta_{2}+\theta_{1}+1$. Then by Theorem 2.2 (b), $C_{0} \cap H_{2}$ consists of a plane, a line and a point. Since $\left|\left(C_{0} \cap H_{2}\right) \cap H_{1}\right|=\left|\left(C_{0} \cap H_{1}\right) \cap \Delta^{\prime}\right|=\theta_{2}+1$, it holds that $\left(C_{0} \cap H_{1}\right) \cap \Delta^{\prime}$ consists of a plane $\delta^{\prime}$ and a point $P^{\prime}$. Then $\left|\left(C_{0} \cap H_{1}\right) \cap l_{1}\right| \geq\left|\delta^{\prime} \cap l_{1}\right| \geq 1$, which is a contradiction to the choice of $l_{1}$. Thus Claim is proved.

By Claim, $C_{0} \cap H_{1}$ contains a 3 -flat, say $\Delta_{1}$. Let $S=\left(C_{0} \cap H_{1}\right)-\Delta_{1}$. We note $|S|=\theta_{1}+q$. On the other hand, since $c_{0}(\Delta) \geq \theta_{2}+1$ for any 3-flat $\Delta$ in $H_{1},|S \cap \Delta| \geq 1$ for any 3 -flat $\Delta \neq \Delta_{1}$ in $H_{1}$. Thus $S$ can be considered as 1-fold blocking set with respect to hyperplanes in $A G(4, q)$. By Theorem 2.1, we have $|S| \geq(4+1-1)(q-1)+1=4 q-3$ which is a contradiction since $q \geq 4$.

Case 3. There is no 4-flat $H$ with $2 \theta_{2}+\theta_{1}+q \leq c_{0}(H) \leq \theta_{3}+\theta_{1}$.

Proof. Suppose that there exists a 4-flat $H_{1}$ with $c_{0}\left(H_{1}\right)=\theta_{3}+$ $\theta_{1}-e q-f, 0 \leq f \leq q-1,0 \leq e \leq q^{2}-q-3$. By (3.4), we have $c_{0}(\Delta) \geq \theta_{2}-e$. Suppose there is a 3 -flat $\Delta_{1}$ with $c_{0}\left(\Delta_{1}\right)=\theta_{2}-e$. Since $0 \leq e \leq q^{2}-q-3$, we have $2 \theta_{1}+2 \leq c_{0}\left(\Delta_{1}\right) \leq \theta_{2}$. By (3.1), we note that $c_{0}(H) \geq \theta_{2}+\theta_{1}+2$ for any 4 -flat $H$ containing $\Delta_{1}$. Thus we have

$$
\begin{aligned}
c_{0} & =c_{0}\left(H_{1}\right)+\sum_{\Delta_{1} \subseteq H, H \neq H_{1}} c_{0}(H)-q c_{0}\left(\Delta_{1}\right) \\
& \geq c_{0}\left(H_{1}\right)+q\left(\theta_{2}+\theta_{1}+2\right)-q\left(\theta_{2}-e\right)=\theta_{3}+\theta_{2}+2 q+\theta_{1}-(f+1),
\end{aligned}
$$

which is a contradiction since $0 \leq f \leq q-1$. Thus $c_{0}(\Delta) \geq \theta_{2}-e+1$ for any 3 -flat $\Delta$ in $H_{1}$.

On the other hand, $H_{1}$ corresponds to an $\left[n_{1}, 5, d_{1}\right]_{q}$ linear code with $n_{1}=\theta_{4}-c_{0}\left(H_{1}\right)=\theta_{4}-\theta_{3}-\theta_{1}+e q+f$ and $d_{1} \geq q^{4}-q^{3}-q+e q+f-e$.

Applying the Griesmer bound, we have

$$
\begin{aligned}
g_{q}\left(5, d_{1}\right) \geq & q^{4}-q^{3}-q+e q+f-e \\
& +q^{3}-q^{2}-1+e+\left\lceil\frac{f-e}{q}\right\rceil \\
& +q^{2}-q+\left\lceil\frac{e q-q+f-e}{q^{2}}\right\rceil
\end{aligned}
$$




$$
\begin{aligned}
& +q-1+\left\lceil\frac{e q-q+f-e}{q^{3}}\right\rceil \\
& +1 \\
& \geq n_{1}+\left\lceil\frac{f-e}{q}\right\rceil+\left\lceil\frac{e q-q+f-e}{q^{2}}\right\rceil+\left\lceil\frac{e q-q+f-e}{q^{3}}\right\rceil .
\end{aligned}
$$

Let

$$
T=\left\lceil\frac{f-e}{q}\right\rceil+\left\lceil\frac{e q-q+f-e}{q^{2}}\right\rceil+\left\lceil\frac{e q-q+f-e}{q^{3}}\right\rceil .
$$

Then $T \leq 0$ by the Griesmer bound. Now we prove the following claim.

Claim. In the set of pairs $(e, f)$ with $0 \leq e \leq q^{2}-q-3$ and $0 \leq f \leq$ $q-1$, we have the following:

$$
T \leq 0 \text { if and only if }(e, f)=(0,0),(1,0) \text {, or }(1,1) .
$$

Moreover, in this case $T=0$.

Proof of Claim: We prove

$$
\begin{cases}T=0, & \text { if }(e, f)=(0,0),(1,0), \text { or }(1,1), \\ T>0, & \text { otherwise. }\end{cases}
$$

When $(e, f)=(0,0),(1,0)$ or $(1,1)$, we note that $T=0$. Hence we consider the other case.

For $f \geq 2$, since $0 \leq e \leq q^{2}-q-3<q^{2}$, we have

$$
\begin{aligned}
T & \geq\left\lceil\frac{f-e}{q}+\frac{e q-q+f-e}{q^{2}}+\frac{e q-q+f-e}{q^{3}}\right\rceil \\
& =\left\lceil\frac{(f-1) \theta_{2}+1-e}{q^{3}}\right\rceil>0 .
\end{aligned}
$$

Now, consider the case $f=0$ or 1 .

Assume $f=1$. We have $e q-q+f-e=(e-1)(q-1)$. For $e=0$, we have $T=\left\lceil\frac{1}{q}\right\rceil+\left\lceil\frac{-(q-1)}{q^{2}}\right\rceil+\left\lceil\frac{-(q-1)}{q^{3}}\right\rceil=1>0$. For $2 \leq e \leq q$, we have $T=\left\lceil\frac{1-e}{q}\right\rceil+\left\lceil\frac{(e-1)(q-1)}{q^{2}}\right\rceil+\left\lceil\frac{(e-1)(q-1)}{q^{3}}\right\rceil=0+1+1=2>0$. For $t q+1 \leq e \leq(t+1) q$ with $1 \leq t \leq q-2$, we have $\left\lceil\frac{1-e}{q}\right\rceil=-t$, $t \leq\left\lceil\frac{(e-1)(q-1)}{q^{2}}\right\rceil \leq t+1$ and $\left\lceil\frac{(e-1)(q-1)}{q^{3}}\right\rceil=1$, and hence $T>0$.

Finally, assume $f=0$. For $2 \leq e \leq q-1$, we get $T=\left\lceil\frac{-e}{q}\right\rceil+$ $\left\lceil\frac{e(q-1)-q}{q^{2}}\right\rceil+\left\lceil\frac{e(q-1)-q}{q^{3}}\right\rceil=0+1+1=2>0$. For $t q \leq e \leq(t+1) q-1$ 
with $1 \leq t \leq q-2$, we have $\left\lceil\frac{-e}{q}\right\rceil=-t, t \leq\left\lceil\frac{e(q-1)-q}{q^{2}}\right\rceil \leq t+1$ and $\left\lceil\frac{e(q-1)-q}{q^{3}}\right\rceil=1$, and hence $T>0$. Thus the claim is proved.

By the claim, there remain only three possibilities for $c_{0}\left(H_{1}\right)$.

If $(e, f)=(0,0)$, then $C_{0} \cap H_{1}$ is a $\left\{\theta_{3}+\theta_{1}, \theta_{2}+1 ; 4, q\right\}$-minihyper, which is impossible by Theorem 2.2 (c).

Assume $(e, f)=(1,0)$. Then $C_{0} \cap H_{1}$ is a $\left\{\theta_{3}+1, \theta_{2} ; 4, q\right\}$-minihyper. By Theorem $2.2(\mathrm{~b})$, the minihyper $C_{0} \cap H_{1}$ consists of a 3 -flat $\Delta_{1}$ and a point $P_{1}$. From $c_{0}=c_{0}\left(H_{1}\right)+\sum_{\Delta_{1} \subseteq H, H \neq H_{1}} c_{0}(H)-q c_{0}\left(\Delta_{1}\right)$, since $c_{0}\left(\Delta_{1}\right)=\theta_{3}$, we have

$$
\sum_{\Delta_{1} \subseteq H, H \neq H_{1}} c_{0}(H)=q\left(\theta_{3}+\theta_{1}+2\right) .
$$

Hence there is a 4-flat $H$ containing $\Delta_{1}$ with $c_{0}(H) \geq \theta_{3}+\theta_{1}+2$, which contradicts Case 1 and Case 2.

If $(e, f)=(1,1)$ then $C_{0} \cap H_{1}$ is a $\left\{\theta_{3}, \theta_{2} ; 4, q\right\}$-minihyper, i.e., a 3 -flat. Then $c_{0}\left(H_{0} \cap H_{1}\right)=\theta_{2}$ or $\theta_{3}$, which is a contradiction by (3.1).

Case 4. There is no 4-flat $H$ with $2 \theta_{2}+\theta_{1} \leq c_{0}(H) \leq 2 \theta_{2}+2 q$.

Proof. Suppose that there exists a 4-flat $H_{1}$ with $c_{0}\left(H_{1}\right)=2 \theta_{2}+$ $2 q-f, 0 \leq f \leq q-1$. For any 3 -flat $\Delta$ in $H_{1}$, by (3.4) we have $c_{0}(\Delta) \geq 2 \theta_{1}+1$.

Suppose that $c_{0}(\Delta) \geq 2 \theta_{1}+2$ for all $\Delta \subseteq H_{1}$. Then $H_{1}$ is an $\left[n_{1}, 5, d_{1}\right]_{q}$ code with $n_{1}=\theta_{4}-2 \theta_{2}-2 q+f$ and $d_{1} \geq q^{4}-2 q^{2}-2 q+f+2$, which contradicts the Griesmer bound. Thus there is a 3-flat $\Delta$ in $H_{1}$ with $c_{0}(\Delta)=2 \theta_{1}+1$. Then $C_{0} \cap H_{1}$ is a $\left\{2 \theta_{2}+2 q-f, 2 \theta_{1}+1 ; 4, q\right\}$ minihyper, which contradicts Theorem 2.5.

Case 5. There is no 4 -flat $H$ with $2 \theta_{2}+1 \leq c_{0}(H) \leq 2 \theta_{2}+q$.

Proof. Suppose that there exists a 4 -flat $H_{1}$ with $c_{0}\left(H_{1}\right)=2 \theta_{2}+q-f$, $0 \leq f \leq q-1$. For any 3 -flat $\Delta$ in $H_{1}$, we have $c_{0}(\Delta) \geq 2 \theta_{1}$ by (3.4). Suppose that $c_{0}(\Delta) \geq 2 \theta_{1}+1$ for any $\Delta$ in $H_{1}$. Then $H_{1}$ corresponds to an $\left[n_{1}, 5, d_{1}\right]_{q}$ linear code with $n_{1}=\theta_{4}-2 \theta_{2}-q+f$ and $d_{1} \geq$ $q^{4}-2 q^{2}-q+f+1$, which contradicts the Griesmer bound. Thus there exists a 3 -flat $\Delta$ in $H_{1}$ with $c_{0}(\Delta)=2 \theta_{1}$, and hence $C_{0} \cap H_{1}$ is a $\left\{2 \theta_{2}+q-f, 2 \theta_{1} ; 4, q\right\}$-minihyper. For $1 \leq f \leq q-1$, by Theorem 2.4, such a minihyper $C_{0} \cap H_{1}$ does not exist. Thus we have $f=0$ and 
$C_{0} \cap H_{1}$ is a $\left\{2 \theta_{2}+q, 2 \theta_{1} ; 4, q\right\}$-minihyper. Let $\Delta_{1}$ be a 3 -flat in $H_{1}$ with $c_{0}\left(\Delta_{1}\right)=2 \theta_{1}$. Then we have

$$
\begin{aligned}
c_{0} & =c_{0}\left(H_{1}\right)+\sum_{\Delta_{1} \subseteq H, H \neq H_{1}} c_{0}(H)-q c_{0}\left(\Delta_{1}\right) \\
& =2 \theta_{2}+q+\sum_{\Delta_{1} \subseteq H, H \neq H_{1}} c_{0}(H)-q \cdot 2 \theta_{1},
\end{aligned}
$$

and hence

$$
\sum_{\Delta_{1} \subseteq H, H \neq H_{1}} c_{0}(H)=q\left(\theta_{2}+\theta_{1}+1\right) .
$$

Thus we conclude $c_{0}(H)=\theta_{2}+\theta_{1}+1$ for all 4 -flat $H\left(\neq H_{1}\right)$ containing $\Delta_{1}$, say $H_{2}, \ldots, H_{q+1}$. By Theorem 2.2 (b), for $2 \leq i \leq q+1, H_{i} \cap C_{0}$ consists of a plane $\delta_{i}$, a line $l_{i}$ and a point $Q_{i}$, respectively. Let $C_{0} \cap \Delta_{1}=$ $C_{0} \cap H_{1} \cap H_{i}=l_{i} \cup m_{i}, 2 \leq i \leq q+1$, where $m_{i}=\delta_{i} \cap H_{1}$ is a line. Since $c_{0}\left(\Delta_{1}\right)=2 \theta_{1}$ and $q \geq 4$, there exist $H_{i}$ and $H_{j}$ such that $m_{i}=m_{j}$, $2 \leq i<j \leq q+1$. Then we note that $\delta_{i} \cap \delta_{j}=m_{i}$. Consider the linear span of $\delta_{i}$ and $\delta_{j}$, denoted by $\left\langle\delta_{i}, \delta_{j}\right\rangle$. Then $c_{0}\left(\left\langle\delta_{i}, \delta_{j}\right\rangle\right) \geq \theta_{2}+q^{2}$. Thus we have

$$
\begin{aligned}
c_{0} & =\sum_{\left\langle\delta_{i}, \delta_{j}\right\rangle \subseteq H} c_{0}(H)-q c_{0}\left(\left\langle\delta_{i}, \delta_{j}\right\rangle\right) \\
& \leq \sum_{\left\langle\delta_{i}, \delta_{j}\right\rangle \subseteq H} c_{0}(H)-q\left(\theta_{2}+q^{2}\right),
\end{aligned}
$$

and hence $\sum_{\left\langle\delta_{i}, \delta_{j}\right\rangle \subseteq H} c_{0}(H) \geq 3 \theta_{3}+2 q-1$. Therefore, there exists a 4-flat $H$ containing $\left\langle\delta_{i}, \delta_{j}\right\rangle$ with $c_{0}(H) \geq 3 q^{2}+5$. However, from Case $1,2,3,4$, there is no 4 -flat $H$ with $2 \theta_{2}+\theta_{1} \leq c_{0}(H) \leq \theta_{3}+2 \theta_{1}+q-1$. Thus we have a contradiction since $q \geq 4$.

Step II. By Step I and (2) we conclude that

$$
\theta_{2}+\theta_{1}+1 \leq c_{0}(H) \leq 2 \theta_{2} \text { for any 4-flat } H \text { in } P G(5, q) .
$$

On the other hand, since $H_{0} \cap C_{0}$ is a disjoint union of a plane $\delta_{0}$, a line $l_{0}$ and a point $P_{0}$, the linear span $\Delta_{0}=\left\langle\delta_{0}, P_{0}\right\rangle$ is 3 -flat with $c_{0}\left(\Delta_{0}\right)=\theta_{2}+2$. Then we have 


$$
\begin{aligned}
c_{0} & =c_{0}\left(H_{0}\right)+\sum_{\Delta_{0} \subseteq H, H \neq H_{0}} c_{0}(H)-q c_{0}\left(\Delta_{0}\right) \\
& =\theta_{2}+\theta_{1}+1+\sum_{\Delta_{0} \subseteq H, H \neq H_{0}} c_{0}(H)-q\left(\theta_{2}+2\right),
\end{aligned}
$$

and hence $\sum_{\Delta_{0} \subseteq H, H \neq H_{0}} c_{0}(H)=2 \theta_{3}+3 q-3$. Thus there is a 4 -flat $H\left(\neq H_{0}\right)$ containing $\Delta_{0}$ with $c_{0}(H) \geq 2 \theta_{2}+3$, which contradicts $(3.5)$. Thus the proof is completed.

\section{References}

[1] A. A. Bruen, Polynomial multiplicities over finite fields and intersection sets, Journal of Combinatorial Theroy, Series A, 60 (1992), 19-33.

[2] E. J. Cheon, T. Kato, S. J. Kim, Nonexistence of $[n, 5, d]_{q}$ codes attaining the Griesmer bound for $q^{4}-2 q^{2}-2 q+1 \leq d \leq q^{4}-2 q^{2}-q$, Designs, Codes and Cryptography, 36 (2005), 289-299.

[3] E. J. Cheon, T. Maruta, On the minimum length of some linear codes, Designs, Codes and Cryptography, 43 (2007), 123-135.

[4] E. J. Cheon, T. Kato, On the minimum length of some linear codes of dimension 6, Bull. Korean Math. Soc., 45 (2008), 419-425.

[5] E. J. Cheon, The non-existence of Griesmer codes with parameters close to codes of Belov type, Designs, Codes and Cryptography, 61 (2011), 131-139.

[6] M. Grassl, Bounds on linear codes $[n, k, d]$ over $G F(q)$, available on line: http://www.codetables.de/

[7] N. Hamada., A characterization of some $[n, k, d ; q]$-codes meeting the Griesmer bound using a minihyper in a finite projective geometry, Discrete Math. 116 (1993), 229-268.

[8] N. Hamada., A survey of recent work on characterization of minihypers in $P G(t, q)$ and nonbinary linear codes meeting the Griesmer bound, J. Combin. Inform. \& Syst. Sci. 18 (1993), 161-191.

[9] R. Hill, Optimal linear codes, Cryptography and Coding II (ed. C. Mitchell), Oxford Univ. Press, Oxford, 75-104 (1992).

[10] A. Klein, K. Metsch, Parameters for which the Griesmer bound is not sharp, Discrete Math., 307 (2007), 2695-2703.

[11] G. Markus, Code Tables: Bounds on the parameters of various types of codes. available on line: http://www.codetables.de/

[12] I. N. Landjev, T. Maruta, On the minimum length of quaternary linear codes of dimension five, Discrete Math., 202 (1999), 145-161.

[13] Maruta T., A characterization of some minihypers and its application to linear codes, Geometriae Dedicata 74 (1999), 305-311.

[14] T. Maruta, On the nonexistence of q-ary linear codes of dimension five, Designs, Codes and Cryptography 22 (2001), 165-177. 
[15] T. Maruta, I. N. Landjev, A. Rousseva, On the minimum size of some minihypers and related linear codes, Designs, Codes and Cryptography, 34 (2005), $5-15$.

[16] T. Maruta, Griesmer Bound for Linear Codes over Finite Fields. available on line: http://www.mi.s.osakafu-u.ac.jp/ ${ }^{\sim}$ maruta/griesmer.htm

[17] T. Maruta, Y. Yoshida, Ternary linear codes and quadrics, Electronic Journal of Combinatorics, 16, \#R9 (2009).

$*$

Department of Mathematics and RINS

Gyeongsang National University

Jinju 660-701, Republic of Korea

E-mail: enju1000@naver.com 